EPJ Web of Conferences 43, 02005 (2013)

DOI: $10.1051 /$ epjconf/20134302005

(C) Owned by the authors, published by EDP Sciences, 2013

\title{
The inner Galactic globular clusters
}

\author{
J. Alonso-García ${ }^{1,2, a}$, M. Catelan ${ }^{1,2}$, M. Mateo ${ }^{3}$ and D. Minniti ${ }^{1,2,4}$ \\ 1 Pontificia Universidad Católica de Chile, Santiago, Chile \\ 2 The Milky Way Millennium Nucleus, Santiago, Chile \\ 3 University of Michigan, Ann Arbor, MI, USA \\ 4 Vatican Observatory, Vatican City State, Italy
}

\begin{abstract}
Galactic globular clusters located towards the inner regions of the Milky Way have been historically neglected, mainly due to the difficulties caused by the presence of an elevated extinction by foreground dust, and high field star densities along the lines of sight where most of them lie. To overcome these difficulties we have developed a new method to map the differential extinction suffered by these clusters, which was successfully applied to a sample of moderately-extincted, luminous, extended, inner Galactic globular clusters observed in the optical, for which we have been able to determine more accurate physical parameters. For the most extincted inner Galactic globular clusters, near-infrared wavelengths provide a more suitable window for their study. The VVV survey, which is currently observing the central regions of the Milky Way at these wavelengths, will provide a comprehensive view, from the inner regions out to their tidal radii and beyond, of most of these globular clusters.
\end{abstract}

\section{INTRODUCTION}

Galactic globular clusters (GCs) are formed by old populations of stars [1]. Although in the last years we have come to believe that their stellar populations were not as simple as it was once thought [2-5], their member stars were born in a short time interval and have, in most cases, not substantial variations in their iron and heavy elements content. This makes Galactic GCs excellent laboratories to test our ideas and theories of stellar evolution. We can also fairly easily obtain their main physical parameters such as age, distance, extinction and metallicity, by comparing their observed color-magnitude diagrams (CMDs) with theoretical models and isochrones, if we have deep CMDs like the ones available for lowly-extincted, outer halo GCs [6]. But in the inner regions of the Milky Way, observations are more complicated to obtain [7]. Extinction, both absolute and differential, is significant due to the amount of gas and dust when we observe towards these low-latitude lines of sight. In addition, the high densities of field stars in these regions make the population of cluster stars dominant only in the central regions of the $\mathrm{GC}$, where the crowding is more significant.

In order to be able to extract physical parameters from the Galactic bulge and inner halo GC population we can follow two different approaches, according to the amount of extinction affecting the GC. Deep observations of GCs that suffer low or moderate extinction are more easily performed at optical wavelengths. However, observations may still suffer the effects of differential extinction. In Section 2 we show a way to deal with this problem and to obtain accurate relative ages, distances and absolute extinctions for these GCs. For the most extincted GC, we need to move to near-infrared wavelengths, as we show in Section 3.

\footnotetext{
ae-mail: jalonso@astro.puc.edu
}

This is an Open Access article distributed under the terms of the Creative Commons Attribution License 2.0, which permits unrestricted use, distribution, and reproduction in any medium, provided the original work is properly cited. 


\section{EPJ Web of Conferences}

\section{INNER GALACTIC GCS IN THE OPTICAL}

The use of medium-sized telescopes with wide-field cameras allows to observe and resolve the crowded inner Galactic fields, and obtain accurate, deep, optical photometry for the GCs that suffer only low or moderate extinction. But the presence of differential extinction complicates the analysis of their CMDs. Recently we have developed a method that allows to map the foreground differential extinction and to eliminate its effects in order to produce cleaned, deep CMDs for the GCs [8]. The method is comprised of five iterative steps:

- We assign a probability to the observed stars to belong to the GCs, based on their positions in the sky and in the CMD. Empirical King models [9] and Besançon models [10] for the sample of GCs are involved in the assignments of these probabilities.

- We produce a ridgeline for the GC in its CMD, using a non-parametric univariate regression of the color as a function of magnitude. We give higher weight to the information from stars with more precise photometry and higher probability of being GC members.

- Stars are moved along the reddening vector until they intersect the ridgeline produced in the previous step, and this way, a color excess is assigned to every observed star.

- These individual extinctions are smoothed over the field of view (FOV) using a non-parametric bivariate regression, generating an extinction map.

- The extinction map is used to differentially deredden the photometry and produce a new cleaned CMD.

We repeat these steps until there is a convergence in the ridgeline of the GC.

We observed a sample of 25 GCs in the bulge and inner halo of the Galaxy, using the Magellan $6.5 \mathrm{~m}$ telescope and the Hubble Space Telescope [11]. The GCs in this sample were chosen to be located towards the inner regions of the Galaxy, to be moderately extincted and sufficiently extended, so that they present a certain degree of differential extinction over their FOV, and to be sufficiently luminous and nearby so that the number of GC stars in the different evolutionary sequences is significant over the FOV. So that we can reach the main sequence with good photometric precision at the turn-off point. We successfully applied the dereddening technique to map the extinction in front of the GCs, and to clean their CMDs (Figure 1, [11]). We compared our data with the theoretical models provided by the Dartmouth library [12] to obtain a new set of precise values for the ages, distances, and absolute reddenings for the sample of GCs. We will detail the comparison and results in a paper that we are currently preparing. In general, we obtained a good agreement between our values of the absolute extinction and the ones found in the literature, provided in the 2003 version of the Harris catalog [13], and provided in the SFD extinction maps [14]. However, we noticed differences increased for the highest extincted sample of GCs, where the SFD maps seem to overestimate the color excesses. A comparison of the distances to the clusters obtained from the isochrone fitting with the ones provided in the Harris catalog shows, in general, a good agreement at small extinctions, but the clusters of the sample with the highest absolute extinction $(E(B-V)>0.4)$ and, maybe more importantly, the highest differential extinction (e.g., NGC 6266, NGC 6273, NGC 6553) are, according to our analysis, $\sim 1 \mathrm{kpc}$ closer to the Sun and $\sim 1 \mathrm{kpc}$ further from the Galactic center. Finally, the inner Galactic GCs in our sample are found to be $\sim 13 \mathrm{Gyr}$ old, and all formed in a short time spread, less than $\sim 1.5 \mathrm{Gyr}$.

\section{INNER GALACTIC GCS IN THE NEAR-INFRARED}

The most extincted GCs found in the Milky Way can suffer dimming in their magnitudes by as much as $A_{V} \sim 10$ or more. This makes deep optical observations of the stellar populations of these objects nearly impossible, even with the best telescopes available. Fortunately, extinction is highly diminished at infrared wavelengths $\left(A_{K} \sim 0.1 A_{V}\right)$. Therefore, we can make use of near-infrared facilities to observe 
Ageing Low Mass Stars: From Red Giants to White Dwarfs
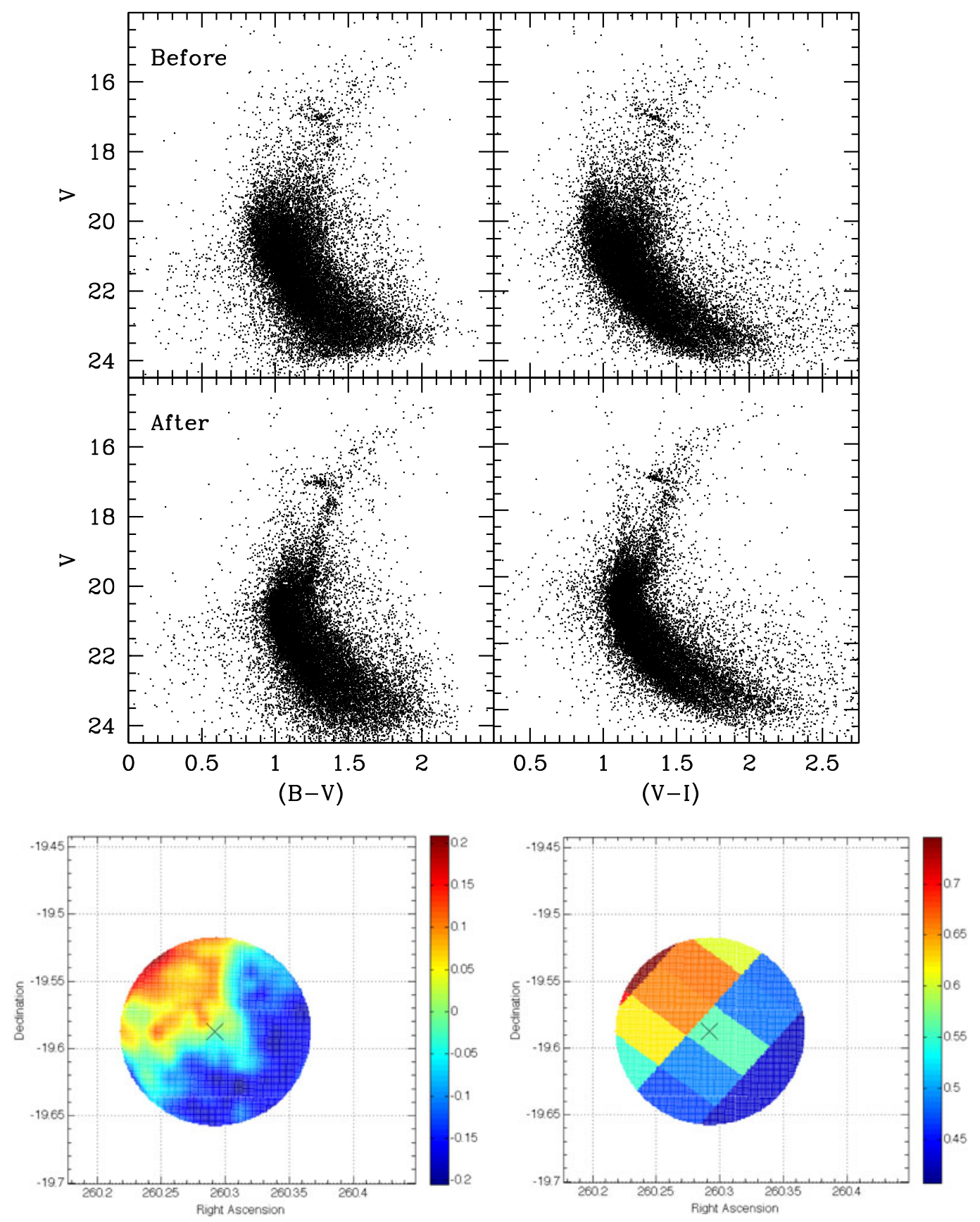

Figure 1. Optical CMDs are shown in the upper panels for NGC6342, one of the clusters in our sample, before and after applying our dereddening technique. Note that the evolutionary sequences get narrower by a factor of 2, which greatly facilitates the comparison with theoretical models. An important by-product of the dereddening process is an extinction map across the GC field (bottom left panel). The overall agreement with the SFD extinction map (bottom right panel) is reasonably good, though a fair comparison requires that we lower the resolution of our maps to match that of the dust maps. The $\times$ symbol marks the position of the GC center. The difference in the color scales is due to the fact that our map is just a differential extinction map, and the adopted reddening zero point, defined by where our ridgeline lies, has to be established from other methods. Adapted from [11]. 

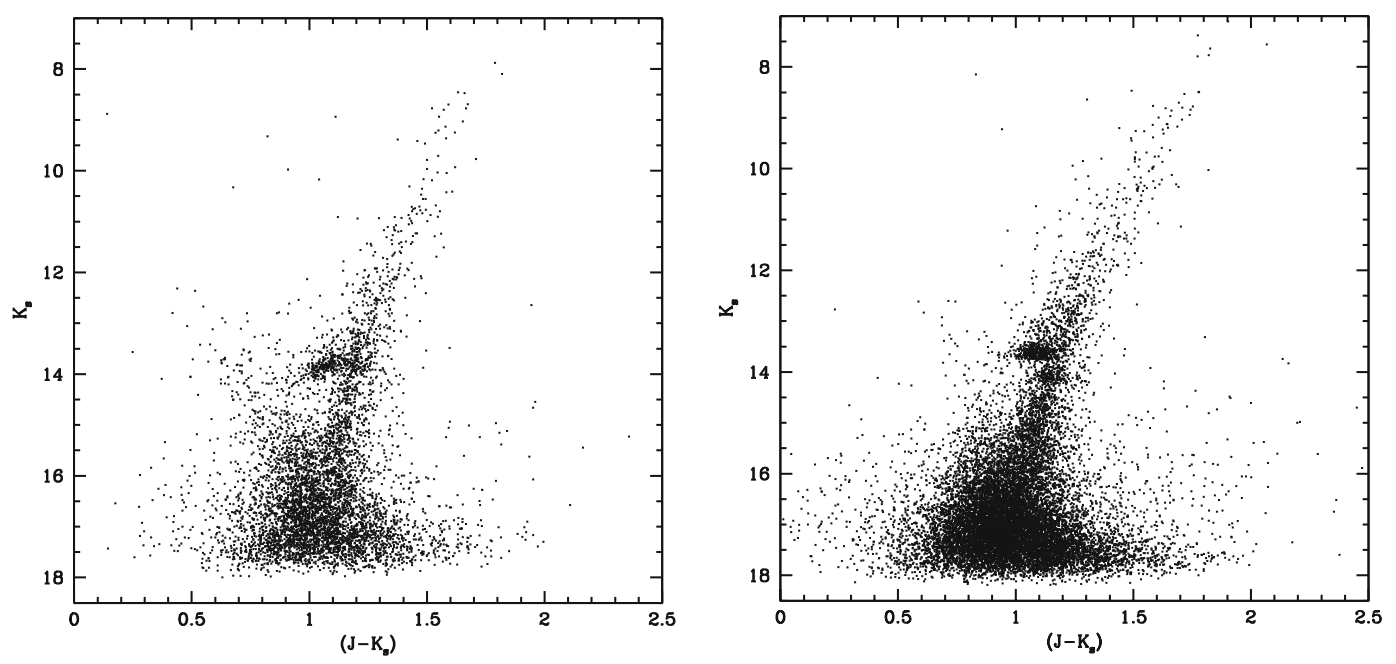

Figure 2. Near-infrared CMDs of NGC 6380 (left) and NGC 6440 (right), two of the GCs that lie in the region surveyed by VVV, presented as an example of the kind of precise near-infrared CMDs which are being obtained from VVV observations.

the most extincted GCs located towards the central parts of the Milky Way and study their stellar populations.

The Vista Variables in the Via Lactea (VVV) near-infrared survey is a fundamental tool in this study. VVV [15-18] is one of the five ESO public surveys currently conducted with the 4m VISTA (Visible and Infrared Survey Telescope for Astronomy) telescope in Cerro Paranal, Chile. VVV surveys an inner region of the Milky Way of $562 \mathrm{deg}^{2}$, that encompasses most of the Galactic bulge $\left(-10.0^{\circ}<l<+10.5^{\circ}\right.$ and $\left.-10.3^{\circ}<b<+5.1^{\circ}\right)$ and an inner Galactic disc region with a high star formation rate $\left(-65.3^{\circ}<l<-10.0^{\circ}\right.$ and $\left.-2.25^{\circ}<b<+2.25^{\circ}\right)$. The VVV survey has produced a deep atlas of the central regions of our Galaxy in five filters in the near-infrared $\left(Z, Y, J, H, K_{s}\right)$ and will produce a catalog of light curves in the $K_{s}$ filter for all objects located towards these regions (see [19], for a first report on variability in the VVV database). Observations are being conducted using the VIRCAM camera at the VISTA telescope, which has a resolution of $0.34^{\prime \prime}$ per pixel and a field of view of $1.48 \times 1.11 \mathrm{deg}^{2}$.

In the region surveyed by VVV there are 37 known Galactic GCs, according to the 2010 version of the Harris catalog [13], plus a few new candidates (e.g., [20, 21]). To extract the best possible photometry from these very crowded fields, we performed PSF photometry in the VVV images using an updated version of DoPHOT [11,22]. The photometry was calibrated by comparison with 2MASS data. We produced the near-infrared CMDs of these objects (see Figure 2), which will be compared in a future work with the latest theoretical models. Although we cannot reach the turn-off point with enough resolution in most cases, we can still use the information provided by the most evolved and brightest regions of the CMDs (red giant branch, asymptotic giant branch and horizontal branch) to extract some of the main physical parameters of these GCs. Interestingly, the complete spatial coverage of the GCs, from the center to their tidal radius and beyond, will allow us to explore any radial gradients of the stellar populations, and provide a better view of the structural parameters of the inner Galactic GCs.

This study was supported by grant 0206081 from NSF, by grant GO10573.01-A from STScI, by the Chilean Ministry for the Economy, Development, and Tourism's Programa Iniciativa Científica Milenio through grant P07021-F, awarded to The Milky Way Millennium Nucleus, by Proyecto Fondecyt Regular 1110326, by Basal CATA PFB-06, and by Anillos ACT-86. 
Ageing Low Mass Stars: From Red Giants to White Dwarfs

\section{References}

[1] Marín-Franch, A., et al. 2009, ApJ, 694, 1498

[2] Piotto, G., et al. 2007, ApJ, 661, L53

[3] Carretta, E., et al. 2009, A\&A, 505, 117

[4] Valcarce, A. A. R. \& Catelan, M. 2011, A\&A, 533, A120

[5] Gratton, R. G., Carretta, E., \& Bragaglia, A. 2012, A\&AR, 20, 50

[6] Alcaino, G., Liller, W., \& Alvarado, F., AJ, 114, 2626

[7] Valenti, E., Ferraro, F. R., \& Origlia, L. 2007, AJ, 133, 1287

[8] Alonso-García, J., Mateo, M., Sen, B., Banerjee, M., \& von Braun, K. 2011, AJ, 141, 146

[9] King, I. 1962, AJ, 67, 471

[10] Robin, A. C., Reylé, C., Derriére, S., \& Picaud, S. 2003, A\&A, 409, 523

[11] Alonso-García, J., Mateo, M., Sen, B., Banerjee, M., Catelan, M., Minniti, D., \& von Braun, K. 2012 AJ, 143, 70

[12] Dotter, A., Chaboyer, B., Jevremovic, D., Baron E., Ferguson, J. W., Sarajedini, A., \& Anderson, J. 2007, AJ, 134, 376

[13] Harris, W.E. 1996, AJ, 112, 1487

[14] Schlegel, D., Finkbeiner, D., \& Davis, M. 1998, ApJ, 500, 525

[15] Minniti, D. et al. 2010, New Astronomy, 15, 44

[16] Saito, R. K, et al. 2010, The Messenger, 141, 24

[17] Catelan, M., et al. 2011, in Carnegie Observatories Astrophysics Series, Vol. 5, RR Lyrae Stars, Metal-Poor Stars, and the Galaxy, ed. A. McWilliam (Pasadena: Carnegie Observatories), 144

[18] Saito, R. K, et al. 2012, A\&A, 537, 107

[19] Dékány, I., et al. 2011, arXiv1111.0909D

[20] Minniti, D., et al. 2011, A\&A, 527, A81

[21] Moni Bidin, C., et al. 2011, A\&A, 535, A33

[22] Schechter, P., Mateo, M., \& Saha, A. 1993, PASP, 105, 1342 